\title{
ANÁLISE FAUNÍSTICA DE LEPIDÓPTEROS EM COMUNIDADES NATURAIS NO MUNICÍPIO DE COTRIGUAÇU-MT, BRASIL
}

\author{
Aline Bispo Santos Januário ${ }^{1}$, Otávio Peres Filho ${ }^{2}$, Alberto Dorval ${ }^{2}$, Marcelo Dias Souza ${ }^{3}$, \\ Marcelo Muniz Silva ${ }^{1}$
}

${ }^{1}$ Programa de Pós-graduação em Ciências Florestais e Ambientais, Universidade Federal de Mato Grosso, Cuiabá, MT, CEP
$78125-070$. E-mail: aline-bis@ bol.com.br; munizflorestal@yahoo.com.br
${ }^{2}$ Faculdade de Engenharia Florestal, Universidade Federal de Mato Grosso, CEP 78125-070. E-mail: peres@ufmt.br;
a.dorval@ hotmail.com
${ }^{3}$ Programa de Pós-graduação em Engenharia Florestal, Universidade Federal do Paraná, CEP 80210-170. E-mail:
marcelo.dias@florestal.eng.br

\section{RESUMO}

Este trabalho teve como objetivo desenvolver estudos faunísticos de lepidópteros em três ambientes florestais: floresta nativa, mata ripária e área de Capoeira no município de Cotriguaçu, MT. Foram instaladas três armadilhas luminosas em cada ambiente. O período de levantamento ocorreu de julho de 2007 a junho de 2008. Foram determinados os índices faunísticos de dominância, frequência, constância e abundância. A diversidade foi calculada pelo índice de Shannon-Wiener (H') e a similaridade dos ambientes foi determinada através da análise de Cluster. Coletou-se um total de 1.558 indivíduos, distribuídos em 236 espécies, da qual ocorreram 170, 113 e 107 respectivamente na floresta nativa, mata ripária e capoeira. Floresta nativa apresentou maior número de espécies coletadas com maior índice de diversidade. Capoeira e mata ripária foram ambientes similares. Nenhuma espécie foi dominante e constante nos três ambientes.

Palavras-chave: armadilha luminosa, levantamento, lepidopterofauna

\section{FAUNISTIC ANALYSIS OF LEPIDOPTERA IN NATURAL COMMUNITIES OF COTRIGUAÇU, STATE OF MATO GROSSO, BRAZIL}

\begin{abstract}
This paper deals with studies of lepidopteran faunal indices in three forest environments: native forest, riparian forest and secondary forest in Cotriguaçu, State of Mato Grosso, Brazil. Three light traps were installed in each environment. The monitoring was done from July 2007 to June 2008. The faunal indices of dominance, frequency, constancy and abundance were determined. The diversity was calculated by Shannon-Wiener $\left(H^{\prime}\right)$ index and the similarity of the environments was determined by Cluster analysis. A total of 1,558 individuals were collected being distributed in 236 species, which occurred 170, 113 and 107, respectively in the native forest, riparian forest, and secondary forest. The native forest showed highest number of species with highest diversity. Secondary forest and riparian forest were similar environments. No species was dominant and constant in all the three environments.
\end{abstract}

Keywords: light trap, survey, monitoring, lepidopterofauna 


\section{INTRODUÇÃO}

Os lepidópteros constituem uma das principais ordens de insetos em número de espécies descritas, com aproximadamente 146.000 espécies descritas. É um grupo que apresenta íntimas associações com seu habitat e grande sensibilidade a mudanças, constituindo-se em indicadoras da qualidade ambiental e integridade de paisagens naturais (MOTTA, 2002).

Atualmente a diversidade biológica vem sendo perdida em ritmo acelerado e a causa principal da redução das populações é a destruição de seu habitat (WOOD \& GILMAN, 1998).

Um dos principais problemas enfrentados, e consequentemente um assunto de muito interesse em todo o mundo, é a perda da diversidade biológica pela degradação de ambiente e destruição de suas populações naturais (FREITAS et al., 2003).

Entre os métodos de amostragem mais empregados para os estudos entomofaunísticos, destaca-se o levantamento através de armadilhas luminosas. Além das coletas de insetos, sua utilização contempla a distribuição e flutuação dos insetos, controle de pragas e análises entomofaunísticas de insetos noturnos fototrópicos positivos (FERREIRA \& MARTINS, 1982).

Em estudos ecológicos, as análises de fauna têm por finalidade a caracterização e a estrutura de comunidades, assim como a avaliação de impactos a partir da comparação de dados de decomposição e abundância de fauna, obtidas em períodos diversos (CAMARGO, 1999; MARINONI et al., 1999).

Com intuito de ampliar os conhecimentos sobre os lepidópteros associados a diferentes comunidades naturais, objetivou-se neste trabalho desenvolver estudos faunísticos em três ambientes florestais, no município de Cotriguaçu, estado de Mato Grosso.

\section{MATERIAL E MÉTODOS}

O estudo foi realizado na fazenda São Nicolau, propriedade da Organización Nacionale du Fôret/Peugeot - ONF Brasil, localizada na região amazônica do noroeste do estado de Mato Grosso, no município de Cotriguaçu, de julho de 2007 a junho de 2008. O clima de acordo com a classificação de Köppen, é o tropical chuvoso. O tipo climático é o "Am", que intercala pequeno período de seca e chuvas inferiores a $60 \mathrm{~mm}$ no mês mais seco. As temperaturas médias anuais são elevadas durante o ano, oscilando entre $23^{\circ}$ e $25^{\circ} \mathrm{C}$, sendo os meses da primavera-verão, os mais quentes e com precipitação média anual de $2.300 \mathrm{~mm}$ (BRASIL, 1982).

A área total da fazenda é de 10.134,43 ha, sendo 2.907,55 ha de área desmatada (floresta nativa que foi substituída por pastagem entre 1974 e 1997), 2.000 ha reservados para o reflorestamento, 294,14 ha como área remanescente e 6.932,74 ha formados por floresta nativa, constituindo a reserva legal da propriedade, onde os ambientes estudados foram: 1) área de Capoeira; 2) Mata Ripária; 3) Floresta Nativa.

Nas coletas foram empregadas armadilhas luminosas semelhantes ao modelo "Luiz de Queiroz" (SILVEIRA NETO \& SILVEIRA, 1969), adaptada com lâmpadas luz do dia fluorescente de 15 watts e 100 volts, baterias automotivas como fonte de energia, sendo diferenciadas apenas pela colocação de uma sacola plástica (saco de lixo de 100 litros com papel picado que dificulta a fuga dos lepidópteros) (FERREIRA \& MARTINS, 1982). As armadilhas foram instaladas a 1,5 metros do solo em suporte de madeira denominado "forca", acionadas por baterias automotivas das 18:00 às 6:00h.

Foram amostrados ambientes de mata ripária (mata ciliar), área de capoeira 
(floresta secundária) e Floresta nativa (área de reserva legal), sendo distribuídas três armadilhas por ambiente. As coletas foram quinzenais e totalizadas mensalmente para efeito de análise. O material coletado foi acondicionado em sacos plásticos etiquetados com a data e identificação da armadilha e enviados ao Laboratório de Proteção Florestal - LAPROFLOR, da Faculdade de Engenharia Florestal da Universidade Federal de Mato Grosso UFMT e mantidos em freezer.

Os lepidópteros coletados foram triados e devidamente quantificados, codificados, registrados de acordo com a armadilha e data de coleta e secos em estufa no laboratório a $60^{\circ} \mathrm{C}$ por 72 horas.

Posteriormente, os indivíduos foram montados e encaminhados ao Prof. Dr. Sinval Silveira Neto, da Escola Superior de Agricultura "Luiz de Queiroz" (ESALQ), da Universidade de São Paulo (USP), para identificação e como suporte comparativo utilizou-se a coleção entomológica do Museu da ESALQ/USP. Os exemplares coletados foram identificados ao nível de família, gênero e quando possível de espécie e encontram-se depositados na coleção entomológica do LAPROFLOR/UFMT.

Dentro de cada ambiente foram realizados estudos faunísticos de dominância de Sakagami \& Laroca (1967), dominância, frequência, constância e abundância de Silveira Neto et al. (1976). A diversidade foi calculada pelo índice de Shannon-Wiener $\left(\mathrm{H}^{\prime}\right)$. Adicionalmente, utilizou-se o exponencial desse índice para comparar a magnitude da diferença de diversidade dos ambientes amostrados, cujo valor é equivalente à diversidade de Hill de primeira ordem: $\mathrm{N} 1=\exp \left(\mathrm{H}^{\prime}\right)$, que expressa $\mathrm{o}$ resultado em termos de número efetivo de espécies (HILL, 1973; JOST, 2006).

Para verificar o grau de similaridade entre os ambientes estudados foi realizada análise de Cluster pelo método da distância média (Unweighted Pair Groups Method using Arithmetic Averages - UPGMA), utilizando o programa STATISTIC 7.0.

\section{RESULTADOS E DISCUSSÃO}

Foram coletados 1.558 indivíduos, sendo 747 na floresta nativa, 463 na mata ripária e 348 indivíduos em área de capoeira, distribuídos em 170, 113 e 107 espécies, respectivamente (Tabela 1). Segundo Price (1975) o nível de qualidade dos recursos tem influência no número de espécies suportadas, enquanto a quantidade dos recursos afeta o número da população de cada espécie. De acordo com o princípio de Thienemann apud Dajoz (1973), quando as condições do meio são favoráveis encontram-se numerosas espécies, sendo cada uma delas representada por um pequeno número de indivíduos, resultando num índice de diversidade elevado; quando as condições são desfavoráveis, encontra-se um pequeno número de espécies, mas cada uma delas é, em geral, representada por numerosos exemplares, sendo, neste caso, pequeno o índice de diversidade.

Devido à tendência atual de preservar parte dos remanescentes florestais nativos e outros recursos naturais, aliada à criação de corredores naturais entremeando povoamentos florestais e ligando fragmentos nativos isolados, podem ser esperados menores problemas com agentes daninhos, principalmente lepidópteros desfolhadores. Esta iniciativa permite a diversificação do estrato vegetal, tornando o ecossistema mais heterogêneo, o que propicia a proliferação e a manutenção de inimigos naturais (SANTOS et al., 2002). 
Tabela 1. Análise faunística das espécies coletadas em floresta nativa, mata ripária e capoeira no município de Cotriguaçu-MT.

\begin{tabular}{|c|c|c|c|c|c|c|c|c|c|c|c|c|c|c|c|}
\hline \multirow{2}{*}{ Família/Gênero/Espécie } & \multicolumn{5}{|c|}{ Floresta nativa } & \multicolumn{5}{|c|}{ Mata ripária } & \multicolumn{5}{|c|}{ Capoeira } \\
\hline & $\begin{array}{c}\mathrm{N}^{\circ} \mathrm{de} \\
\text { Indivíduos }\end{array}$ & $\mathrm{D}$ & $\mathrm{A}$ & $\mathrm{F}$ & $\mathrm{C}$ & $\begin{array}{c}\mathrm{N}^{\circ} \text { de } \\
\text { Indivíduos }\end{array}$ & $\mathrm{D}$ & A & $\mathrm{F}$ & $\mathrm{C}$ & $\begin{array}{c}\mathrm{N}^{\circ} \text { de } \\
\text { Indivíduos }\end{array}$ & $\mathrm{D}$ & $\mathrm{A}$ & $\mathrm{F}$ & $\mathrm{C}$ \\
\hline \multicolumn{16}{|l|}{ Bombycidae } \\
\hline Apatelodes pandara & 1 & nd & $\mathrm{r}$ & $\mathrm{pf}$ & $\mathrm{z}$ & 1 & nd & $\mathrm{r}$ & $\mathrm{pf}$ & $\mathrm{Z}$ & - & - & - & - & - \\
\hline Olceclostera nina & 1 & nd & $\mathrm{r}$ & $\mathrm{pf}$ & $\mathrm{z}$ & - & - & - & - & - & - & - & - & - & - \\
\hline \multicolumn{16}{|l|}{ Cossidae } \\
\hline Morpheis pyracmon & - & - & - & - & - & - & - & - & - & - & 1 & nd & $\mathrm{r}$ & $\mathrm{pf}$ & $\mathrm{z}$ \\
\hline \multicolumn{16}{|l|}{ Crambidae } \\
\hline Agathodes designalis & - & - & - & - & - & 1 & nd & $\mathrm{r}$ & $\mathrm{pf}$ & $\mathrm{z}$ & 3 & nd & $\mathrm{c}$ & $\mathrm{f}$ & $\mathrm{z}$ \\
\hline Diaphania hyalinata & 2 & nd & $\mathrm{r}$ & $\mathrm{pf}$ & $\mathrm{z}$ & - & - & - & - & - & 1 & nd & $\mathrm{r}$ & $\mathrm{pf}$ & $\mathrm{z}$ \\
\hline Dichogama redtenbacheri & - & - & - & - & - & - & - & - & - & - & 1 & nd & $\mathrm{r}$ & $\mathrm{pf}$ & $\mathrm{z}$ \\
\hline Hyalea sp. & 1 & nd & $\mathrm{r}$ & $\mathrm{pf}$ & $\mathrm{z}$ & 1 & nd & $\mathrm{r}$ & $\mathrm{pf}$ & $\mathrm{z}$ & - & - & - & - & - \\
\hline Loxostege sp.1 & 1 & nd & $\mathrm{r}$ & $\mathrm{pf}$ & $\mathrm{z}$ & - & - & - & - & - & 2 & nd & $\mathrm{c}$ & $\mathrm{pf}$ & $\mathrm{z}$ \\
\hline Loxostege sp.2 & 28 & $\mathrm{~d}$ & $\mathrm{ma}$ & $\mathrm{mf}$ & $\mathrm{y}$ & 41 & $\mathrm{~d}$ & $\mathrm{ma}$ & $\mathrm{mf}$ & $\mathrm{y}$ & 47 & $\mathrm{~d}$ & $\mathrm{ma}$ & $\mathrm{mf}$ & $\mathrm{y}$ \\
\hline Maruca vitrata & 70 & d & $\mathrm{ma}$ & $\mathrm{mf}$ & $\mathrm{w}$ & 63 & $\mathrm{~d}$ & $\mathrm{ma}$ & $\mathrm{mf}$ & $\mathrm{w}$ & 38 & $\mathrm{~d}$ & $\mathrm{ma}$ & $\mathrm{mf}$ & $\mathrm{y}$ \\
\hline Noorda esmeralda & 5 & $\mathrm{~d}$ & $\mathrm{c}$ & $f$ & $\mathrm{y}$ & 5 & $\mathrm{~d}$ & $\mathrm{c}$ & $\mathrm{f}$ & $\mathrm{z}$ & 1 & nd & $\mathrm{r}$ & $\mathrm{pf}$ & $\mathrm{z}$ \\
\hline Omiodes indicatus & 2 & nd & $\mathrm{r}$ & $\mathrm{pf}$ & $\mathrm{z}$ & 17 & $\mathrm{~d}$ & $\mathrm{ma}$ & $\mathrm{mf}$ & $\mathrm{z}$ & - & - & - & - & - \\
\hline Palpita quadristigmalis & 1 & nd & $\mathrm{r}$ & $\mathrm{pf}$ & $\mathrm{Z}$ & - & - & - & - & - & 1 & nd & $\mathrm{r}$ & $\mathrm{pf}$ & $\mathrm{z}$ \\
\hline Polygrammodes ostrealis & - & - & - & - & - & 1 & nd & $\mathrm{r}$ & $\mathrm{pf}$ & $\mathrm{z}$ & - & - & - & - & - \\
\hline Phostria tedea & 2 & nd & $\mathrm{r}$ & $\mathrm{pf}$ & $\mathrm{z}$ & - & - & - & - & - & 1 & nd & $\mathrm{r}$ & $\mathrm{pf}$ & $\mathrm{z}$ \\
\hline Samea ecclesialis & 5 & $\mathrm{~d}$ & $\mathrm{c}$ & f & $\mathrm{Z}$ & 18 & $\mathrm{~d}$ & $\mathrm{ma}$ & $\mathrm{mf}$ & $\mathrm{Z}$ & 2 & nd & $\mathrm{c}$ & $\mathrm{f}$ & $\mathrm{z}$ \\
\hline Samea sp. & 4 & nd & $\mathrm{c}$ & $\mathrm{f}$ & $\mathrm{z}$ & - & - & - & - & - & - & - & - & - & - \\
\hline Siga liris & 1 & nd & $\mathrm{r}$ & $\mathrm{pf}$ & $\mathrm{z}$ & 2 & nd & $\mathrm{d}$ & $\mathrm{pf}$ & $\mathrm{z}$ & 1 & nd & $\mathrm{r}$ & $\mathrm{pf}$ & $\mathrm{z}$ \\
\hline Syngamia florella & 1 & nd & $\mathrm{r}$ & $\mathrm{pf}$ & $\mathrm{Z}$ & 1 & nd & $\mathrm{r}$ & $\mathrm{pf}$ & $\mathrm{Z}$ & 1 & nd & $\mathrm{r}$ & $\mathrm{pf}$ & $\mathrm{z}$ \\
\hline Zinckenia fascialis & - & - & - & - & - & - & - & - & - & - & 1 & nd & $\mathrm{r}$ & $\mathrm{pf}$ & $\mathrm{z}$ \\
\hline
\end{tabular}

D: Dominância - (sd) super dominante; (d) dominante; (nd) não dominante. A: Abundância - (sa) super abundante; (ma) muito abundante; (a) abundante; (c) comum; (d) dispersa; (r) rara. F: Frequência - (sf) super frequente; (mf) muito frequente; (f) frequente; (pf) pouco frequente. C: Constância - (w) constante; (y) acessória; (z) acidental. 
Análise faunística de lepidópteros em comunidades naturais no município de Cotriguaçu-MT, Brasil

\begin{tabular}{|c|c|c|c|c|c|c|c|c|c|c|c|c|c|c|c|}
\hline \multirow{2}{*}{$\begin{array}{l}\text { Família/Gênero/ } \\
\text { Espécie }\end{array}$} & \multicolumn{5}{|c|}{ Floresta nativa } & \multicolumn{5}{|c|}{ Mata ripária } & \multicolumn{5}{|c|}{ Capoeira } \\
\hline & $\begin{array}{c}\mathrm{N}^{\circ} \mathrm{de} \\
\text { Indivíduos } \\
\end{array}$ & $\mathrm{D}$ & A & $\mathrm{F}$ & $\mathrm{C}$ & $\begin{array}{c}\mathrm{N}^{\circ} \mathrm{de} \\
\text { Indivíduos }\end{array}$ & $\mathrm{D}$ & A & $\mathrm{F}$ & $\mathrm{C}$ & $\begin{array}{c}\mathrm{N}^{\circ} \mathrm{de} \\
\text { Indivíduos }\end{array}$ & $\mathrm{D}$ & A & $\mathrm{F}$ & $\mathrm{C}$ \\
\hline \multicolumn{16}{|l|}{ Erebidae } \\
\hline Aclytia heber & 2 & nd & $\mathrm{r}$ & $\mathrm{pf}$ & $\mathrm{z}$ & 3 & nd & $\mathrm{c}$ & $\mathrm{f}$ & $\mathrm{Z}$ & - & - & - & - & - \\
\hline Ammalo sp. & 3 & nd & $\mathrm{c}$ & $\mathrm{f}$ & $\mathrm{z}$ & - & - & - & - & - & - & - & - & - & - \\
\hline Anticarsia gemmatalis & 2 & nd & $\mathrm{r}$ & $\mathrm{pf}$ & $\mathrm{z}$ & - & - & - & - & - & 1 & nd & $\mathrm{r}$ & $\mathrm{pf}$ & $\mathrm{z}$ \\
\hline Argidia sp.1 & 1 & nd & $\mathrm{r}$ & $\mathrm{pf}$ & $\mathrm{z}$ & 2 & nd & $\mathrm{r}$ & $\mathrm{pf}$ & $\mathrm{z}$ & 4 & $\mathrm{~d}$ & $\mathrm{c}$ & $\mathrm{f}$ & $\mathrm{z}$ \\
\hline Argidia sp.2 & 4 & nd & $\mathrm{c}$ & $\mathrm{f}$ & $\mathrm{y}$ & - & - & - & - & - & 1 & nd & $\mathrm{r}$ & $\mathrm{pf}$ & $\mathrm{z}$ \\
\hline Argidia sp. 3 & 1 & nd & $\mathrm{r}$ & $\mathrm{pf}$ & $\mathrm{z}$ & 1 & nd & $\mathrm{r}$ & $\mathrm{pf}$ & $\mathrm{z}$ & - & - & - & - & - \\
\hline Ascalapha odorata & 1 & nd & $\mathrm{r}$ & $\mathrm{pf}$ & $\mathrm{z}$ & - & - & - & - & - & - & - & - & - & - \\
\hline Baniana sp. & 4 & nd & $\mathrm{c}$ & $\mathrm{f}$ & $\mathrm{z}$ & - & - & - & - & - & - & - & - & - & - \\
\hline Belemnia inaurata & 4 & nd & c & $\mathrm{f}$ & $\mathrm{y}$ & 1 & nd & $\mathrm{r}$ & $\mathrm{pf}$ & $\mathrm{z}$ & - & - & - & - & - \\
\hline Bleptina confusalis & 1 & nd & $\mathrm{r}$ & $\mathrm{pf}$ & $\mathrm{z}$ & - & - & - & - & - & - & - & - & - & - \\
\hline Calyptis semicuprea & - & - & - & - & - & 1 & nd & $\mathrm{r}$ & $\mathrm{pf}$ & $\mathrm{z}$ & - & - & - & - & - \\
\hline Ceroctena amynta & 2 & nd & $\mathrm{r}$ & $\mathrm{pf}$ & $\mathrm{z}$ & - & - & - & - & - & - & - & - & - & - \\
\hline Coenipeta sp. & 3 & nd & $\mathrm{c}$ & $\mathrm{f}$ & $\mathrm{z}$ & 1 & nd & $\mathrm{r}$ & $\mathrm{pf}$ & $\mathrm{z}$ & 1 & nd & $\mathrm{r}$ & $\mathrm{pf}$ & $\mathrm{z}$ \\
\hline Cosmosoma sp. & 14 & $\mathrm{~d}$ & $\mathrm{ma}$ & $\mathrm{mf}$ & $\mathrm{y}$ & 3 & nd & $\mathrm{c}$ & $\mathrm{f}$ & $\mathrm{z}$ & 2 & nd & $\mathrm{c}$ & $\mathrm{f}$ & $\mathrm{z}$ \\
\hline Cydosia rimata & 2 & nd & $\mathrm{r}$ & $\mathrm{pf}$ & $\mathrm{z}$ & 1 & nd & $\mathrm{r}$ & $\mathrm{pf}$ & $\mathrm{z}$ & - & - & - & - & - \\
\hline Dycladia lucetius & - & - & - & - & - & - & - & - & - & - & 1 & nd & $\mathrm{r}$ & $\mathrm{pf}$ & $\mathrm{z}$ \\
\hline Dysschema sacrifice & 1 & nd & $\mathrm{r}$ & $\mathrm{pf}$ & $\mathrm{z}$ & 2 & nd & $\mathrm{d}$ & $\mathrm{pf}$ & $\mathrm{z}$ & - & - & - & - & - \\
\hline Dysschema sp.1 & - & - & - & - & - & - & - & - & - & - & 1 & nd & $\mathrm{r}$ & $\mathrm{pf}$ & $\mathrm{z}$ \\
\hline Dysschema sp. 2 & - & - & - & - & - & - & - & - & - & - & 2 & nd & $\mathrm{c}$ & $\mathrm{f}$ & $\mathrm{z}$ \\
\hline Dysschema sp.3 & 1 & nd & $\mathrm{r}$ & $\mathrm{pf}$ & $\mathrm{z}$ & - & - & - & - & - & - & - & - & - & - \\
\hline Dysschema subapicalis & - & - & - & - & - & 1 & nd & $\mathrm{r}$ & $\mathrm{pf}$ & $\mathrm{Z}$ & - & - & - & - & - \\
\hline Epidesma sp. & - & - & - & - & - & - & - & - & - & - & 1 & nd & $\mathrm{r}$ & $\mathrm{pf}$ & $\mathrm{z}$ \\
\hline Epitausa coppryi & 7 & $\mathrm{~d}$ & $\mathrm{ma}$ & $\mathrm{mf}$ & $\mathrm{y}$ & 2 & nd & $\mathrm{r}$ & $\mathrm{pf}$ & $\mathrm{Z}$ & 7 & $\mathrm{~d}$ & $\mathrm{ma}$ & $\mathrm{mf}$ & $\mathrm{y}$ \\
\hline
\end{tabular}

D: Dominância - (sd) super dominante; (d) dominante; (nd) não dominante. A: Abundância - (sa) super abundante; (ma) muito abundante; (a) abundante; (c) comum; (d) dispersa; (r) rara. F: Frequência - (sf) super frequente; (mf) muito frequente; (f) frequente; (pf) pouco frequente. C: Constância - (w) constante; (y) acessória; (z) acidental.

Continua... 


\begin{tabular}{|c|c|c|c|c|c|c|c|c|c|c|c|c|c|c|c|}
\hline \multirow{2}{*}{$\begin{array}{l}\text { Família/Gênero/ } \\
\text { Espécie }\end{array}$} & \multicolumn{5}{|c|}{ Floresta nativa } & \multicolumn{5}{|c|}{ Mata ripária } & \multicolumn{5}{|c|}{ Capoeira } \\
\hline & $\begin{array}{c}\mathrm{N}^{\circ} \mathrm{de} \\
\text { Indivíduos }\end{array}$ & $\mathrm{D}$ & A & $\mathrm{F}$ & $\mathrm{C}$ & $\begin{array}{c}\mathrm{N}^{\circ} \mathrm{de} \\
\text { Indivíduos }\end{array}$ & $\mathrm{D}$ & A & $\mathrm{F}$ & $\mathrm{C}$ & $\begin{array}{c}\mathrm{N}^{\circ} \mathrm{de} \\
\text { Indivíduos }\end{array}$ & $\mathrm{D}$ & $\mathrm{A}$ & $\mathrm{F}$ & $\mathrm{C}$ \\
\hline Eublemma sp. & 12 & $\mathrm{~d}$ & $\mathrm{ma}$ & $\mathrm{mf}$ & $\mathrm{y}$ & 5 & nd & $r$ & $\mathrm{pf}$ & $\mathrm{Z}$ & 6 & $\mathrm{~d}$ & $\mathrm{ma}$ & $\mathrm{mf}$ & $\mathrm{z}$ \\
\hline Eucereon rosa & 1 & nd & $\mathrm{r}$ & $\mathrm{pf}$ & $\mathrm{z}$ & - & - & - & - & - & 2 & nd & $\mathrm{c}$ & $\mathrm{f}$ & $\mathrm{z}$ \\
\hline Eucereon sp. & 4 & nd & $\mathrm{c}$ & $\mathrm{f}$ & $\mathrm{y}$ & 4 & nd & $\mathrm{c}$ & $\mathrm{f}$ & $\mathrm{y}$ & 1 & nd & $\mathrm{r}$ & $\mathrm{pf}$ & $\mathrm{z}$ \\
\hline Eulepidotis detracta & 1 & nd & $\mathrm{r}$ & $\mathrm{pf}$ & $\mathrm{z}$ & - & - & - & - & - & - & - & - & - & - \\
\hline Eulepidotis sp.1 & 1 & nd & $\mathrm{r}$ & $\mathrm{pf}$ & $\mathrm{z}$ & - & - & - & - & - & - & - & - & - & - \\
\hline Eulepidotis sp. 2 & 2 & nd & $\mathrm{r}$ & $\mathrm{pf}$ & $\mathrm{z}$ & 1 & nd & $\mathrm{r}$ & $\mathrm{pf}$ & $\mathrm{z}$ & - & - & - & - & - \\
\hline Eulepidotis sp.3 & 2 & nd & $\mathrm{r}$ & $\mathrm{pf}$ & $\mathrm{z}$ & 12 & nd & $\mathrm{r}$ & $\mathrm{pf}$ & $\mathrm{z}$ & 7 & $\mathrm{~d}$ & $\mathrm{ma}$ & $\mathrm{mf}$ & $\mathrm{z}$ \\
\hline Evius albicoxae & - & - & - & - & - & 1 & nd & $\mathrm{r}$ & $\mathrm{pf}$ & $\mathrm{z}$ & - & - & - & - & - \\
\hline Gonodonta sicheas & 1 & nd & $\mathrm{r}$ & $\mathrm{pf}$ & $\mathrm{z}$ & - & - & - & - & - & - & - & - & - & - \\
\hline Hemeroblemma sp. & 1 & nd & $\mathrm{r}$ & $\mathrm{pf}$ & $\mathrm{z}$ & - & - & - & - & - & 1 & nd & $\mathrm{r}$ & $\mathrm{pf}$ & $\mathrm{z}$ \\
\hline Hyalurga sp.1 & 1 & nd & $\mathrm{r}$ & pf & $\mathrm{z}$ & - & - & - & - & - & - & - & - & - & - \\
\hline Hyalurga sp. 2 & - & - & - & - & - & - & - & - & - & - & 1 & nd & $\mathrm{r}$ & $\mathrm{pf}$ & $\mathrm{z}$ \\
\hline Hyalurga sp.3 & 3 & nd & $\mathrm{c}$ & $\mathrm{f}$ & $\mathrm{y}$ & - & - & - & - & - & - & - & - & - & - \\
\hline Hyalurga syma & 1 & nd & $\mathrm{r}$ & $\mathrm{pf}$ & $\mathrm{z}$ & - & - & - & - & - & - & - & - & - & - \\
\hline Hypena sp. & - & - & - & - & - & - & - & - & - & - & 1 & nd & $\mathrm{r}$ & $\mathrm{pf}$ & $\mathrm{z}$ \\
\hline Hypercompe sp. & - & - & - & - & - & - & - & - & - & - & 1 & nd & $\mathrm{r}$ & $\mathrm{pf}$ & $\mathrm{z}$ \\
\hline Hyponerita tipolis & 5 & $\mathrm{~d}$ & $\mathrm{c}$ & $\mathrm{f}$ & $\mathrm{z}$ & 1 & nd & $\mathrm{r}$ & $\mathrm{pf}$ & $\mathrm{z}$ & 1 & nd & $\mathrm{r}$ & $\mathrm{pf}$ & $\mathrm{z}$ \\
\hline Idalus herois & - & - & - & - & - & - & - & - & - & - & 1 & nd & $\mathrm{r}$ & $\mathrm{pf}$ & $\mathrm{z}$ \\
\hline Lesmone formularis & - & - & - & - & - & - & - & - & - & - & 1 & nd & $\mathrm{r}$ & $\mathrm{pf}$ & $\mathrm{z}$ \\
\hline Letis sp.1 & - & - & - & - & - & 1 & nd & $\mathrm{r}$ & $\mathrm{pf}$ & $\mathrm{z}$ & - & - & - & - & - \\
\hline Letis sp. 2 & - & - & - & - & - & - & - & - & - & - & 1 & nd & $\mathrm{r}$ & $\mathrm{pf}$ & $\mathrm{z}$ \\
\hline Leucanopsis sp. & 2 & nd & $\mathrm{r}$ & $\mathrm{pf}$ & $\mathrm{z}$ & 1 & nd & $\mathrm{r}$ & $\mathrm{pf}$ & $\mathrm{z}$ & - & - & - & - & - \\
\hline Massala hieroglyphica & 2 & nd & $\mathrm{r}$ & $\mathrm{pf}$ & $\mathrm{z}$ & - & - & - & - & - & - & - & - & - & - \\
\hline Melese sp.1 & 2 & nd & $\mathrm{r}$ & $\mathrm{pf}$ & $\mathrm{z}$ & 1 & nd & $\mathrm{r}$ & pf & $\mathrm{z}$ & - & - & - & - & - \\
\hline
\end{tabular}
comum; (d) dispersa; (r) rara. F: Frequência - (sf) super frequente; (mf) muito frequente; (f) frequente; (pf) pouco frequente. C: Constância - (w) constante; (y) acessória; (z) acidental. 
Análise faunística de lepidópteros em comunidades naturais no município de Cotriguaçu-MT, Brasil

\begin{tabular}{|c|c|c|c|c|c|c|c|c|c|c|c|c|c|c|c|}
\hline \multirow{2}{*}{$\begin{array}{l}\text { Família/Gênero/ } \\
\text { Espécie }\end{array}$} & \multicolumn{5}{|c|}{ Floresta nativa } & \multicolumn{5}{|c|}{ Mata ripária } & \multicolumn{5}{|c|}{ Capoeira } \\
\hline & $\begin{array}{c}\mathrm{N}^{\circ} \mathrm{de} \\
\text { Indivíduos }\end{array}$ & $\mathrm{D}$ & A & $\mathrm{F}$ & $\mathrm{C}$ & $\begin{array}{c}\mathrm{N}^{\circ} \text { de } \\
\text { Indivíduos }\end{array}$ & $\mathrm{D}$ & $\mathrm{A}$ & $\mathrm{F}$ & $\mathrm{C}$ & $\begin{array}{c}\mathrm{N}^{\circ} \text { de } \\
\text { Indivíduos }\end{array}$ & $\mathrm{D}$ & A & $\mathrm{F}$ & $\mathrm{C}$ \\
\hline Melese sp.2 & 1 & nd & $\mathrm{r}$ & $\mathrm{pf}$ & $\mathrm{z}$ & - & - & - & - & - & - & - & - & - & - \\
\hline Melese sp.3 & 2 & nd & $\mathrm{r}$ & $\mathrm{pf}$ & $\mathrm{Z}$ & 1 & nd & $\mathrm{r}$ & $\mathrm{pf}$ & $\mathrm{z}$ & 3 & nd & $\mathrm{c}$ & $f$ & $\mathrm{y}$ \\
\hline Melipotis perpendicularis & 2 & nd & $\mathrm{r}$ & $\mathrm{pf}$ & $\mathrm{z}$ & 1 & nd & $\mathrm{r}$ & $\mathrm{pf}$ & $\mathrm{z}$ & - & - & - & - & - \\
\hline Melipotis sp. & - & - & - & - & - & 2 & nd & $\mathrm{d}$ & $\mathrm{pf}$ & $\mathrm{z}$ & 1 & nd & $\mathrm{r}$ & $\mathrm{pf}$ & $\mathrm{z}$ \\
\hline Mocis disserverans & 3 & nd & $\mathrm{c}$ & $\mathrm{f}$ & $\mathrm{z}$ & 7 & $\mathrm{~d}$ & $\mathrm{ma}$ & $\mathrm{mf}$ & $\mathrm{z}$ & 5 & $\mathrm{~d}$ & ma & $\mathrm{mf}$ & $\mathrm{z}$ \\
\hline Mocis latipes & 8 & $\mathrm{~d}$ & $\mathrm{ma}$ & $\mathrm{mf}$ & $\mathrm{y}$ & 5 & $\mathrm{~d}$ & $\mathrm{c}$ & $\mathrm{f}$ & $\mathrm{y}$ & 2 & nd & $\mathrm{c}$ & $f$ & $\mathrm{Z}$ \\
\hline Mocis sp. & 1 & nd & $\mathrm{r}$ & $\mathrm{pf}$ & $\mathrm{z}$ & 1 & nd & $\mathrm{r}$ & $\mathrm{pf}$ & $\mathrm{z}$ & - & - & - & - & - \\
\hline Neonerita sp. & - & - & - & - & - & 2 & nd & $\mathrm{d}$ & $\mathrm{pf}$ & $\mathrm{z}$ & 1 & nd & $\mathrm{r}$ & $\mathrm{pf}$ & $\mathrm{z}$ \\
\hline Nodozana fifi & 4 & nd & $\mathrm{c}$ & $\mathrm{f}$ & $\mathrm{y}$ & 1 & nd & $\mathrm{r}$ & $\mathrm{pf}$ & $\mathrm{z}$ & 1 & nd & $\mathrm{r}$ & $\mathrm{pf}$ & $\mathrm{Z}$ \\
\hline Nodozana thricophora & 1 & nd & $\mathrm{r}$ & $\mathrm{pf}$ & $\mathrm{z}$ & - & - & - & - & - & - & - & - & - & - \\
\hline Ophisma troricalis & - & - & - & - & - & 1 & nd & $\mathrm{r}$ & $\mathrm{pf}$ & $\mathrm{z}$ & - & - & - & - & - \\
\hline Paracles paula & - & - & - & - & - & 3 & nd & $\mathrm{c}$ & $\mathrm{f}$ & $\mathrm{z}$ & - & - & - & - & - \\
\hline Paracles sp.1 & 111 & $\mathrm{~d}$ & $\mathrm{ma}$ & $\mathrm{mf}$ & $\mathrm{w}$ & 25 & $\mathrm{~d}$ & $\mathrm{ma}$ & $\mathrm{mf}$ & $\mathrm{y}$ & 25 & $\mathrm{~d}$ & $\mathrm{ma}$ & $\mathrm{mf}$ & $\mathrm{y}$ \\
\hline Paracles sp. 2 & 1 & nd & $\mathrm{r}$ & $\mathrm{pf}$ & $\mathrm{z}$ & - & - & - & - & - & 1 & nd & $\mathrm{r}$ & $\mathrm{pf}$ & $\mathrm{z}$ \\
\hline Paranerita sp.1 & - & - & - & - & - & 1 & nd & $\mathrm{r}$ & $\mathrm{pf}$ & $\mathrm{z}$ & 1 & nd & $\mathrm{r}$ & $\mathrm{pf}$ & $\mathrm{z}$ \\
\hline Paranerita sp.2 & 2 & nd & $\mathrm{r}$ & $\mathrm{pf}$ & $\mathrm{z}$ & - & - & - & - & - & - & - & - & - & - \\
\hline Pararcte sp. & 1 & nd & $\mathrm{r}$ & $\mathrm{pf}$ & $\mathrm{z}$ & - & - & - & - & - & - & - & - & - & - \\
\hline Pareuchaetes insulata & - & - & - & - & - & - & - & - & - & - & 1 & nd & $\mathrm{r}$ & $\mathrm{pf}$ & $\mathrm{Z}$ \\
\hline Perasia sp. 1 & 1 & nd & $\mathrm{r}$ & $\mathrm{pf}$ & $\mathrm{z}$ & - & - & - & - & - & - & - & - & - & - \\
\hline Perasia sp. 2 & 5 & $\mathrm{~d}$ & $\mathrm{c}$ & $f$ & $\mathrm{y}$ & 5 & $\mathrm{~d}$ & $\mathrm{c}$ & $\mathrm{f}$ & $\mathrm{z}$ & 1 & nd & $\mathrm{r}$ & $\mathrm{pf}$ & $\mathrm{Z}$ \\
\hline Psoloptera sp. & - & - & - & - & - & 1 & nd & $\mathrm{r}$ & $\mathrm{pf}$ & $\mathrm{z}$ & - & - & - & - & - \\
\hline Ptichodis sp. & 1 & nd & $\mathrm{r}$ & $\mathrm{pf}$ & $\mathrm{z}$ & 1 & nd & $\mathrm{r}$ & $\mathrm{pf}$ & $\mathrm{z}$ & - & - & - & - & - \\
\hline Sciopsyche sp. & 6 & $\mathrm{~d}$ & $\mathrm{c}$ & $f$ & $\mathrm{z}$ & 1 & nd & $\mathrm{r}$ & $\mathrm{pf}$ & $\mathrm{z}$ & 1 & nd & $\mathrm{r}$ & $\mathrm{pf}$ & $\mathrm{z}$ \\
\hline Thioptera sp. & 2 & nd & $\mathrm{r}$ & $\mathrm{pf}$ & $\mathrm{Z}$ & - & - & - & - & - & - & - & - & - & - \\
\hline
\end{tabular}

D: Dominância - (sd) super dominante; (d) dominante; (nd) não dominante. A: Abundância - (sa) super abundante; (ma) muito abundante; (a) abundante; (c) comum; (d) dispersa; (r) rara. F: Frequência - (sf) super frequente; (mf) muito frequente; (f) frequente; (pf) pouco frequente. C: Constância - (w) constante; (y) acessória; (z) acidental.

Continua... 


\begin{tabular}{|c|c|c|c|c|c|c|c|c|c|c|c|c|c|c|c|}
\hline \multirow{2}{*}{$\begin{array}{l}\text { Família/Gênero/ } \\
\text { Espécie }\end{array}$} & \multicolumn{5}{|c|}{ Floresta nativa } & \multicolumn{5}{|c|}{ Mata ripária } & \multicolumn{5}{|c|}{ Capoeira } \\
\hline & $\begin{array}{c}\mathrm{N}^{\circ} \mathrm{de} \\
\text { Indivíduos }\end{array}$ & $\mathrm{D}$ & A & $\mathrm{F}$ & $\mathrm{C}$ & $\begin{array}{c}\mathrm{N}^{\circ} \mathrm{de} \\
\text { Indivíduos }\end{array}$ & $\mathrm{D}$ & $\mathrm{A}$ & $\mathrm{F}$ & $\mathrm{C}$ & $\begin{array}{c}\mathrm{N}^{\circ} \mathrm{de} \\
\text { Indivíduos }\end{array}$ & $\mathrm{D}$ & A & $\mathrm{F}$ & $\mathrm{C}$ \\
\hline Trichura cerberus & 2 & nd & $\mathrm{r}$ & $\mathrm{pf}$ & $\mathrm{Z}$ & - & - & - & - & - & - & - & - & - & - \\
\hline Xanthopastis timais & - & - & - & - & - & 1 & nd & $\mathrm{r}$ & $\mathrm{pf}$ & $\mathrm{z}$ & - & - & - & - & - \\
\hline Zale sp. & 1 & nd & $\mathrm{r}$ & $\mathrm{pf}$ & $\mathrm{Z}$ & 1 & nd & $\mathrm{r}$ & $\mathrm{pf}$ & $\mathrm{Z}$ & 2 & nd & $\mathrm{c}$ & $\mathrm{f}$ & $\mathrm{z}$ \\
\hline \multicolumn{16}{|l|}{ Geometridae } \\
\hline Epimecis puellaria & 2 & nd & $\mathrm{r}$ & $\mathrm{pf}$ & $\mathrm{z}$ & - & - & - & - & - & 1 & nd & $\mathrm{r}$ & $\mathrm{pf}$ & $\mathrm{z}$ \\
\hline Epimecis sp.1 & - & - & - & - & - & 1 & nd & $\mathrm{r}$ & $\mathrm{pf}$ & $\mathrm{z}$ & 3 & nd & $\mathrm{c}$ & $\mathrm{f}$ & $\mathrm{z}$ \\
\hline Epimecis sp.2 & - & - & - & - & - & 3 & nd & $\mathrm{c}$ & $\mathrm{f}$ & $\mathrm{y}$ & - & - & - & - & - \\
\hline Leuciris minutepunctaria & - & - & - & - & - & - & - & - & - & - & 1 & nd & $\mathrm{r}$ & $\mathrm{pf}$ & $\mathrm{z}$ \\
\hline Oospila sp. & 7 & $\mathrm{~d}$ & $\mathrm{ma}$ & $\mathrm{mf}$ & $\mathrm{z}$ & - & - & - & - & - & 2 & nd & $\mathrm{c}$ & $\mathrm{f}$ & $\mathrm{z}$ \\
\hline Oxydia agliata & 3 & nd & $\mathrm{c}$ & $\mathrm{f}$ & $\mathrm{z}$ & - & - & - & - & - & - & - & - & - & - \\
\hline Oxydia sp.1 & 1 & nd & $\mathrm{r}$ & $\mathrm{pf}$ & $\mathrm{z}$ & - & - & - & - & - & 1 & nd & $\mathrm{r}$ & $\mathrm{pf}$ & $\mathrm{z}$ \\
\hline Oxydia sp. 2 & - & - & - & - & - & 1 & nd & $\mathrm{r}$ & $\mathrm{pf}$ & $\mathrm{z}$ & - & - & - & - & - \\
\hline Oxydia vesulia & - & - & - & - & - & 1 & nd & $\mathrm{r}$ & $\mathrm{pf}$ & $\mathrm{z}$ & - & - & - & - & - \\
\hline Pero amanda & 3 & nd & $\mathrm{c}$ & $\mathrm{f}$ & $\mathrm{y}$ & 1 & nd & $\mathrm{r}$ & $\mathrm{pf}$ & $\mathrm{z}$ & 1 & nd & $\mathrm{r}$ & $\mathrm{pf}$ & $\mathrm{z}$ \\
\hline Pero sp.1 & 1 & nd & $\mathrm{r}$ & $\mathrm{pf}$ & $\mathrm{z}$ & - & - & - & - & - & - & - & - & - & - \\
\hline Pero sp. 2 & 1 & nd & $\mathrm{r}$ & $\mathrm{pf}$ & $\mathrm{z}$ & - & - & - & - & - & - & - & - & - & - \\
\hline Pero sp. 3 & 2 & nd & $\mathrm{r}$ & $\mathrm{pf}$ & $\mathrm{z}$ & - & - & - & - & - & - & - & - & - & - \\
\hline Pero sp.4 & - & - & - & - & - & 1 & nd & $\mathrm{r}$ & $\mathrm{pf}$ & $\mathrm{z}$ & - & - & - & - & - \\
\hline Phrudocentra sp. & 5 & $\mathrm{~d}$ & $\mathrm{c}$ & $\mathrm{f}$ & $\mathrm{y}$ & - & - & - & - & - & 3 & nd & $\mathrm{c}$ & $\mathrm{f}$ & $\mathrm{y}$ \\
\hline Semiothisa sp.1 & 6 & $\mathrm{~d}$ & $\mathrm{c}$ & $\mathrm{f}$ & $\mathrm{z}$ & - & - & - & - & - & - & - & - & - & - \\
\hline Semiothisa sp.2 & 5 & $\mathrm{~d}$ & $\mathrm{c}$ & $\mathrm{f}$ & $\mathrm{y}$ & 4 & nd & $\mathrm{c}$ & $\mathrm{f}$ & $\mathrm{z}$ & - & - & - & - & - \\
\hline Semiothisa sp.3 & 29 & $\mathrm{~d}$ & $\mathrm{ma}$ & $\mathrm{mf}$ & $\mathrm{w}$ & 25 & $\mathrm{~d}$ & $\mathrm{ma}$ & $\mathrm{mf}$ & $\mathrm{y}$ & 19 & $\mathrm{~d}$ & ma & $\mathrm{mf}$ & $\mathrm{w}$ \\
\hline Sphacelodes vulneraria & 1 & nd & $\mathrm{r}$ & $\mathrm{pf}$ & $\mathrm{z}$ & - & - & - & - & - & - & - & - & - & - \\
\hline Synchlora gerularia & 8 & $\mathrm{~d}$ & $\mathrm{ma}$ & $\mathrm{mf}$ & $\mathrm{y}$ & 12 & $\mathrm{~d}$ & $\mathrm{ma}$ & $\mathrm{mf}$ & $\mathrm{y}$ & 13 & $\mathrm{~d}$ & $\mathrm{ma}$ & $\mathrm{mf}$ & $\mathrm{y}$ \\
\hline
\end{tabular}

Continua... 
Análise faunística de lepidópteros em comunidades naturais no município de Cotriguaçu-MT, Brasil

\begin{tabular}{|c|c|c|c|c|c|c|c|c|c|c|c|c|c|c|c|}
\hline \multirow{2}{*}{$\begin{array}{l}\text { Família/Gênero/ } \\
\text { Espécie }\end{array}$} & \multicolumn{5}{|c|}{ Floresta nativa } & \multicolumn{5}{|c|}{ Mata ripária } & \multicolumn{5}{|c|}{ Capoeira } \\
\hline & $\begin{array}{c}\mathrm{N}^{\circ} \mathrm{de} \\
\text { Indivíduos }\end{array}$ & $\mathrm{D}$ & A & $\mathrm{F}$ & $\mathrm{C}$ & $\begin{array}{c}\mathrm{N}^{\circ} \mathrm{de} \\
\text { Indivíduos }\end{array}$ & $\mathrm{D}$ & A & $\mathrm{F}$ & $\mathrm{C}$ & $\begin{array}{c}\mathrm{N}^{\circ} \mathrm{de} \\
\text { Indivíduos }\end{array}$ & $\mathrm{D}$ & A & $\mathrm{F}$ & $\mathrm{C}$ \\
\hline Synchlora sp.1 & 2 & nd & $\mathrm{r}$ & $\mathrm{pf}$ & $\mathrm{z}$ & - & - & - & - & - & 1 & nd & $\mathrm{r}$ & $\mathrm{pf}$ & $\mathrm{Z}$ \\
\hline Synchlora sp.2 & 15 & $\mathrm{~d}$ & $\mathrm{ma}$ & $\mathrm{mf}$ & $\mathrm{w}$ & 7 & $\mathrm{~d}$ & $\mathrm{ma}$ & $\mathrm{mf}$ & $\mathrm{y}$ & 5 & $\mathrm{~d}$ & $\mathrm{ma}$ & $\mathrm{mf}$ & $\mathrm{y}$ \\
\hline Synchlora sp.3 & 1 & nd & $\mathrm{r}$ & $\mathrm{pf}$ & $\mathrm{z}$ & - & - & - & - & - & - & - & - & - & - \\
\hline Synchlora sp.4 & 1 & nd & $\mathrm{r}$ & $\mathrm{pf}$ & $\mathrm{z}$ & - & - & - & - & - & - & - & - & - & - \\
\hline Thyrinteina schadeana & - & - & - & - & - & 4 & nd & c & $\mathrm{f}$ & $\mathrm{z}$ & 1 & nd & $\mathrm{r}$ & $\mathrm{pf}$ & $\mathrm{z}$ \\
\hline \multicolumn{16}{|l|}{ Hedylidae } \\
\hline Macrosoma sp. & 8 & $\mathrm{~d}$ & $\mathrm{ma}$ & $\mathrm{mf}$ & $\mathrm{w}$ & 4 & nd & $\mathrm{c}$ & $\mathrm{f}$ & $\mathrm{y}$ & 3 & nd & c & $\mathrm{f}$ & $\mathrm{z}$ \\
\hline \multicolumn{16}{|l|}{ Hepialidae } \\
\hline Dalaca sp.1 & 1 & nd & $\mathrm{r}$ & $\mathrm{pf}$ & $\mathrm{z}$ & - & - & - & - & - & 2 & nd & $\mathrm{c}$ & $\mathrm{f}$ & $\mathrm{z}$ \\
\hline Dalaca sp.2 & 1 & nd & $\mathrm{r}$ & $\mathrm{pf}$ & $\mathrm{z}$ & - & - & - & - & - & - & - & - & - & - \\
\hline \multicolumn{16}{|l|}{ Hesperiidae } \\
\hline Astraptes fulgerator & 1 & nd & $\mathrm{r}$ & $\mathrm{pf}$ & $\mathrm{z}$ & 1 & nd & $\mathrm{r}$ & $\mathrm{pf}$ & $\mathrm{z}$ & - & - & - & - & - \\
\hline Calpodes ethlius & 4 & nd & $\mathrm{c}$ & $\mathrm{f}$ & $\mathrm{z}$ & - & - & - & - & - & - & - & - & - & - \\
\hline Corticea sp. & 1 & nd & $\mathrm{r}$ & $\mathrm{pf}$ & $\mathrm{z}$ & - & - & - & - & - & - & - & - & - & - \\
\hline Dyscophellus porcius & - & - & - & - & - & 1 & nd & $\mathrm{r}$ & $\mathrm{pf}$ & $\mathrm{z}$ & - & - & - & - & - \\
\hline Epargyreus sp. & 1 & nd & $\mathrm{r}$ & $\mathrm{pf}$ & $\mathrm{z}$ & - & - & - & - & - & - & - & - & - & - \\
\hline Proteides mercurius & - & - & - & - & - & - & - & - & - & - & 1 & nd & $\mathrm{r}$ & pf & $\mathrm{z}$ \\
\hline Pyrrhopyge charybdis & - & - & - & - & - & 1 & nd & $\mathrm{r}$ & $\mathrm{pf}$ & $\mathrm{z}$ & - & - & - & - & - \\
\hline Quinta sp. & - & - & - & - & - & 1 & nd & $\mathrm{r}$ & $\mathrm{pf}$ & $\mathrm{z}$ & - & - & - & - & - \\
\hline Thespieus sp. & 3 & nd & $\mathrm{c}$ & $\mathrm{f}$ & $\mathrm{z}$ & 1 & nd & $\mathrm{r}$ & $\mathrm{pf}$ & $\mathrm{z}$ & - & - & - & - & - \\
\hline Urbanus proteus & 1 & nd & $\mathrm{r}$ & $\mathrm{pf}$ & $\mathrm{z}$ & - & - & - & - & - & - & - & - & - & - \\
\hline Urbanus simplicius & 1 & nd & $\mathrm{r}$ & pf & $\mathrm{z}$ & - & - & - & - & - & - & - & - & - & - \\
\hline
\end{tabular}

D: Dominância - (sd) super dominante; (d) dominante; (nd) não dominante. A: Abundância - (sa) super abundante; (ma) muito abundante; (a) abundante; (c) comum; (d) dispersa; (r) rara. F: Frequência - (sf) super frequente; (mf) muito frequente; (f) frequente; (pf) pouco frequente. C: Constância - (w) constante; (y) acessória; (z) acidental.

Continua... 


\begin{tabular}{|c|c|c|c|c|c|c|c|c|c|c|c|c|c|c|c|}
\hline \multirow{2}{*}{$\begin{array}{l}\text { Família/Gênero/ } \\
\text { Espécie }\end{array}$} & \multicolumn{5}{|c|}{ Floresta nativa } & \multicolumn{5}{|c|}{ Mata ripária } & \multicolumn{5}{|c|}{ Capoeira } \\
\hline & $\begin{array}{c}\mathrm{N}^{\circ} \text { de } \\
\text { Indivíduos }\end{array}$ & $\mathrm{D}$ & A & $\mathrm{F}$ & $\mathrm{C}$ & $\begin{array}{c}\mathrm{N}^{\circ} \mathrm{de} \\
\text { Indivíduos }\end{array}$ & $\mathrm{D}$ & A & $\mathrm{F}$ & $\mathrm{C}$ & $\begin{array}{c}\mathrm{N}^{\circ} \mathrm{de} \\
\text { Indivíduos }\end{array}$ & $\mathrm{D}$ & A & $\mathrm{F}$ & $\mathrm{C}$ \\
\hline \multicolumn{16}{|l|}{ Lasiocampidae } \\
\hline Euglyphis sp.1 & 1 & nd & $\mathrm{r}$ & $\mathrm{pf}$ & $\mathrm{z}$ & - & - & - & - & - & - & - & - & - & - \\
\hline Euglyphis sp.2 & 1 & nd & $\mathrm{r}$ & $\mathrm{pf}$ & $\mathrm{z}$ & 1 & nd & $\mathrm{r}$ & $\mathrm{pf}$ & $\mathrm{z}$ & - & - & - & - & - \\
\hline \multicolumn{16}{|l|}{ Limacodidae } \\
\hline Acharia nesea & 2 & nd & $\mathrm{r}$ & $\mathrm{pf}$ & $\mathrm{z}$ & - & - & - & - & - & - & - & - & - & - \\
\hline Euphobetron moorei & - & - & - & - & - & 1 & nd & $\mathrm{r}$ & $\mathrm{pf}$ & $\mathrm{z}$ & 2 & nd & $\mathrm{c}$ & $\mathrm{f}$ & $\mathrm{z}$ \\
\hline \multicolumn{16}{|l|}{ Lycaenidae } \\
\hline Cyanophrys acaste & 1 & nd & $\mathrm{r}$ & $\mathrm{pf}$ & $\mathrm{z}$ & - & - & - & - & - & - & - & - & - & - \\
\hline Strephonota sp. & 1 & nd & $\mathrm{r}$ & $\mathrm{pf}$ & $\mathrm{z}$ & - & - & - & - & - & - & - & - & - & - \\
\hline \multicolumn{16}{|l|}{ Megalopygidae } \\
\hline Megalopyge lanata & 1 & nd & $\mathrm{r}$ & $\mathrm{pf}$ & $\mathrm{z}$ & 1 & nd & $\mathrm{r}$ & $\mathrm{pf}$ & $\mathrm{Z}$ & - & - & - & - & - \\
\hline Megalopyge sp. & 4 & nd & $\mathrm{c}$ & $\mathrm{f}$ & $\mathrm{y}$ & - & - & - & - & - & - & - & - & - & - \\
\hline Norape plumosa & 12 & $\mathrm{~d}$ & $\mathrm{ma}$ & $\mathrm{mf}$ & $\mathrm{y}$ & 4 & nd & $\mathrm{c}$ & $\mathrm{f}$ & $\mathrm{y}$ & 4 & $\mathrm{~d}$ & $\mathrm{c}$ & $\mathrm{f}$ & $\mathrm{z}$ \\
\hline Podalia sp. & - & - & - & - & - & - & - & - & - & - & - & - & - & - & - \\
\hline Trosia $\mathrm{sp}$. & - & - & - & - & - & - & - & - & - & - & 1 & nd & $\mathrm{r}$ & $\mathrm{pf}$ & $\mathrm{z}$ \\
\hline \multicolumn{16}{|l|}{ Noctuidae } \\
\hline Agrotis repleta & 1 & nd & $\mathrm{r}$ & $\mathrm{pf}$ & $\mathrm{z}$ & - & - & - & - & - & - & - & - & - & - \\
\hline Argyrogramma sp. & - & - & - & - & - & 1 & nd & $\mathrm{c}$ & $\mathrm{f}$ & $\mathrm{Z}$ & 1 & nd & $\mathrm{r}$ & $\mathrm{pf}$ & $\mathrm{z}$ \\
\hline Argyrogramma verruca & 1 & nd & $\mathrm{r}$ & $\mathrm{pf}$ & $\mathrm{z}$ & - & - & - & - & - & - & - & - & - & - \\
\hline Bagisara repanda & 1 & nd & $\mathrm{r}$ & $\mathrm{pf}$ & $\mathrm{z}$ & - & - & - & - & - & - & - & - & - & - \\
\hline Cucullia sp. & 3 & nd & $\mathrm{c}$ & $\mathrm{f}$ & $\mathrm{z}$ & - & - & - & - & - & - & - & - & - & - \\
\hline Elaphria deltoids & 4 & nd & $\mathrm{c}$ & $\mathrm{f}$ & $\mathrm{y}$ & 1 & nd & $\mathrm{r}$ & $\mathrm{pf}$ & $\mathrm{z}$ & 1 & nd & $\mathrm{r}$ & $\mathrm{pf}$ & $\mathrm{Z}$ \\
\hline Elaphria grata & 1 & nd & $\mathrm{r}$ & $\mathrm{pf}$ & $\mathrm{z}$ & - & - & - & - & - & - & - & - & - & - \\
\hline
\end{tabular}

D: Dominância - (sd) super dominante; (d) dominante; (nd) não dominante. A: Abundância - (sa) super abundante; (ma) muito abundante; (a) abundante; (c) comum; (d) dispersa; (r) rara. F: Frequência - (sf) super frequente; (mf) muito frequente; (f) frequente; (pf) pouco frequente. C: Constância - (w) constante; (y) acessória; (z) acidental. 
Análise faunística de lepidópteros em comunidades naturais no município de Cotriguaçu-MT, Brasil

\begin{tabular}{|c|c|c|c|c|c|c|c|c|c|c|c|c|c|c|c|}
\hline \multirow{2}{*}{$\begin{array}{l}\text { Família/Gênero/ } \\
\text { Espécie }\end{array}$} & \multicolumn{5}{|c|}{ Floresta nativa } & \multicolumn{5}{|c|}{ Mata ripária } & \multicolumn{5}{|c|}{ Capoeira } \\
\hline & $\begin{array}{c}\mathrm{N}^{\circ} \mathrm{de} \\
\text { Indivíduos }\end{array}$ & $\mathrm{D}$ & A & $\mathrm{F}$ & $\mathrm{C}$ & $\begin{array}{c}\mathrm{N}^{\circ} \mathrm{de} \\
\text { Indivíduos }\end{array}$ & $\mathrm{D}$ & A & $\mathrm{F}$ & $\mathrm{C}$ & $\begin{array}{c}\mathrm{N}^{\circ} \text { de } \\
\text { Indivíduos }\end{array}$ & $\mathrm{D}$ & A & $\mathrm{F}$ & $\mathrm{C}$ \\
\hline Elaphria sp.1 & - & - & - & - & - & 1 & nd & $\mathrm{r}$ & $\mathrm{pf}$ & $\mathrm{z}$ & - & - & - & - & - \\
\hline Elaphria sp.2 & 1 & nd & $\mathrm{r}$ & $\mathrm{pf}$ & $\mathrm{z}$ & 1 & nd & $\mathrm{r}$ & $\mathrm{pf}$ & $\mathrm{z}$ & - & - & - & - & - \\
\hline Eriopyga sp. & 1 & nd & $\mathrm{r}$ & $\mathrm{pf}$ & $\mathrm{z}$ & - & - & - & - & - & - & - & - & - & - \\
\hline Heliothis virescens & - & - & - & - & - & 1 & nd & $\mathrm{r}$ & $\mathrm{pf}$ & $\mathrm{z}$ & - & - & - & - & - \\
\hline Leucania sp.1 & - & - & - & - & - & 1 & nd & $\mathrm{r}$ & $\mathrm{pf}$ & $\mathrm{z}$ & - & - & - & - & - \\
\hline Leucania sp. 2 & 6 & $\mathrm{~d}$ & $\mathrm{c}$ & $\mathrm{f}$ & $\mathrm{y}$ & 6 & $\mathrm{~d}$ & $\mathrm{a}$ & $\mathrm{mf}$ & $\mathrm{z}$ & 8 & $\mathrm{~d}$ & ma & $\mathrm{mf}$ & $\mathrm{y}$ \\
\hline Leucania sp.3 & 2 & nd & $\mathrm{r}$ & $\mathrm{pf}$ & $\mathrm{z}$ & 1 & nd & $\mathrm{r}$ & $\mathrm{pf}$ & $\mathrm{z}$ & 1 & nd & $\mathrm{r}$ & $\mathrm{pf}$ & $\mathrm{z}$ \\
\hline Leucania striguscala & 4 & nd & $\mathrm{c}$ & $\mathrm{f}$ & $\mathrm{y}$ & 5 & d & $\mathrm{c}$ & $\mathrm{pf}$ & $\mathrm{z}$ & 1 & nd & $\mathrm{r}$ & $\mathrm{pf}$ & $\mathrm{z}$ \\
\hline Pseudoleucania sp. & 1 & nd & $\mathrm{r}$ & $\mathrm{pf}$ & $\mathrm{z}$ & - & - & - & - & - & - & - & - & - & - \\
\hline Spodoptera eridania & - & - & - & - & - & 1 & nd & $\mathrm{r}$ & $\mathrm{pf}$ & $\mathrm{z}$ & - & - & - & - & - \\
\hline Spodoptera frugiperda & - & - & - & - & - & 3 & nd & $\mathrm{c}$ & $\mathrm{f}$ & $\mathrm{z}$ & - & - & - & - & - \\
\hline \multicolumn{16}{|l|}{ Notodontidae } \\
\hline Chliara cresus & 1 & nd & $\mathrm{r}$ & $\mathrm{pf}$ & $\mathrm{z}$ & - & - & - & - & - & - & - & - & - & - \\
\hline Hemiceras sp.1 & - & - & - & - & - & - & - & - & - & - & 1 & nd & $\mathrm{r}$ & $\mathrm{pf}$ & $\mathrm{z}$ \\
\hline Hemiceras sp.2 & 2 & nd & $\mathrm{r}$ & $\mathrm{pf}$ & $\mathrm{z}$ & - & - & - & - & - & 4 & $\mathrm{~d}$ & $\mathrm{c}$ & $\mathrm{f}$ & $\mathrm{z}$ \\
\hline Lepasta grammodes & - & - & - & - & - & - & - & - & - & - & 1 & nd & $\mathrm{r}$ & $\mathrm{pf}$ & $\mathrm{z}$ \\
\hline Lepasta sp. & - & - & - & - & - & 2 & nd & $\mathrm{d}$ & $\mathrm{pf}$ & $\mathrm{z}$ & - & - & - & - & - \\
\hline Lirimiris sp.1 & - & - & - & - & - & - & - & - & - & - & 1 & nd & $\mathrm{r}$ & $\mathrm{pf}$ & $\mathrm{Z}$ \\
\hline Lirimiris sp.2 & 1 & nd & $\mathrm{r}$ & $\mathrm{pf}$ & $\mathrm{z}$ & 3 & nd & $\mathrm{c}$ & $\mathrm{f}$ & $\mathrm{z}$ & 1 & nd & $\mathrm{r}$ & $\mathrm{pf}$ & $\mathrm{z}$ \\
\hline Lirimiris truncata & 1 & nd & $\mathrm{r}$ & $\mathrm{pf}$ & $\mathrm{z}$ & 2 & nd & $\mathrm{d}$ & $\mathrm{pf}$ & $\mathrm{z}$ & - & - & - & - & - \\
\hline Marthula sp. & 1 & nd & $\mathrm{r}$ & $\mathrm{pf}$ & $\mathrm{z}$ & - & - & - & - & - & - & - & - & - & - \\
\hline Rosema demorsa & 6 & $\mathrm{~d}$ & $\mathrm{c}$ & $\mathrm{f}$ & $\mathrm{y}$ & 1 & nd & $\mathrm{r}$ & $\mathrm{pf}$ & $\mathrm{z}$ & 1 & nd & $\mathrm{r}$ & $\mathrm{pf}$ & $\mathrm{z}$ \\
\hline Rosema dorsalis & 7 & $\mathrm{~d}$ & ma & $\mathrm{mf}$ & $\mathrm{y}$ & 1 & nd & $\mathrm{r}$ & $\mathrm{pf}$ & $\mathrm{z}$ & 1 & nd & $\mathrm{r}$ & $\mathrm{pf}$ & $\mathrm{z}$ \\
\hline Rosema sciritis & 5 & $\mathrm{~d}$ & $\mathrm{c}$ & $\mathrm{f}$ & $\mathrm{z}$ & 3 & nd & $\mathrm{c}$ & $\mathrm{f}$ & $\mathrm{z}$ & - & - & - & - & - \\
\hline
\end{tabular}

D: Dominância - (sd) super dominante; (d) dominante; (nd) não dominante. A: Abundância - (sa) super abundante; (ma) muito abundante; (a) abundante; (c) comum; (d) dispersa; (r) rara. F: Frequência - (sf) super frequente; (mf) muito frequente; (f) frequente; (pf) pouco frequente. C: Constância - (w) constante; (y) acessória; (z) acidental.

Continua... 


\begin{tabular}{|c|c|c|c|c|c|c|c|c|c|c|c|c|c|c|c|}
\hline \multirow{2}{*}{$\begin{array}{l}\text { Família/Gênero/ } \\
\text { Espécie }\end{array}$} & \multicolumn{5}{|c|}{ Floresta nativa } & \multicolumn{5}{|c|}{ Mata ripária } & \multicolumn{5}{|c|}{ Capoeira } \\
\hline & $\begin{array}{c}\mathrm{N}^{\circ} \mathrm{de} \\
\text { Indivíduos }\end{array}$ & $\mathrm{D}$ & A & $\mathrm{F}$ & $\mathrm{C}$ & $\begin{array}{c}\mathrm{N}^{\circ} \mathrm{de} \\
\text { Indivíduos }\end{array}$ & $\mathrm{D}$ & A & $\mathrm{F}$ & $\mathrm{C}$ & $\begin{array}{c}\mathrm{N}^{\circ} \mathrm{de} \\
\text { Indivíduos }\end{array}$ & $\mathrm{D}$ & A & $\mathrm{F}$ & $\mathrm{C}$ \\
\hline \multicolumn{16}{|l|}{ Nymphalidae } \\
\hline Adelpha mesentina & 1 & nd & $\mathrm{r}$ & $\mathrm{pf}$ & $\mathrm{Z}$ & - & - & - & - & - & - & - & - & - & - \\
\hline Adelpha sp. & 1 & nd & $\mathrm{r}$ & $\mathrm{pf}$ & $\mathrm{z}$ & - & - & - & - & - & - & - & - & - & - \\
\hline Archeoprepona demophoon & 1 & nd & $\mathrm{r}$ & $\mathrm{pf}$ & $\mathrm{z}$ & 1 & nd & $\mathrm{r}$ & $\mathrm{pf}$ & $\mathrm{Z}$ & - & - & - & - & - \\
\hline Brassolis sophorae & 1 & nd & $\mathrm{r}$ & $\mathrm{pf}$ & $\mathrm{z}$ & - & - & - & - & - & - & - & - & - & - \\
\hline Caligo brasiliensis & 1 & nd & $\mathrm{r}$ & $\mathrm{pf}$ & $\mathrm{Z}$ & - & - & - & - & - & - & - & - & - & - \\
\hline Dasyophthalma sp. & 1 & nd & $\mathrm{r}$ & $\mathrm{pf}$ & $\mathrm{z}$ & - & - & - & - & - & - & - & - & - & - \\
\hline Eunica tatila & 1 & nd & $\mathrm{r}$ & $\mathrm{pf}$ & $\mathrm{z}$ & - & - & - & - & - & - & - & - & - & - \\
\hline Myscelia orsis & - & - & - & - & - & 1 & nd & $\mathrm{r}$ & $\mathrm{pf}$ & $\mathrm{Z}$ & - & - & - & - & - \\
\hline Taygetis sp. & 1 & nd & $\mathrm{r}$ & $\mathrm{pf}$ & $\mathrm{z}$ & - & - & - & - & - & 1 & nd & $\mathrm{r}$ & $\mathrm{pf}$ & $\mathrm{z}$ \\
\hline \multicolumn{16}{|l|}{ Elachistidae } \\
\hline Loxotoma elegans & - & - & - & - & - & - & - & - & - & - & 1 & nd & $\mathrm{r}$ & $\mathrm{pf}$ & $\mathrm{z}$ \\
\hline Stenoma catenifer & 1 & nd & $\mathrm{r}$ & $\mathrm{pf}$ & $\mathrm{z}$ & - & - & - & - & - & 1 & nd & $\mathrm{r}$ & $\mathrm{pf}$ & $\mathrm{z}$ \\
\hline \multicolumn{16}{|l|}{ Pieridae } \\
\hline Aphrissa statira & - & - & - & - & - & 1 & nd & $\mathrm{r}$ & $\mathrm{pf}$ & $\mathrm{z}$ & - & - & - & - & - \\
\hline \multicolumn{16}{|l|}{ Psychidae } \\
\hline Oiketicus kirbyi & - & - & - & - & - & - & - & - & - & - & 1 & nd & $\mathrm{r}$ & $\mathrm{pf}$ & $\mathrm{z}$ \\
\hline \multicolumn{16}{|l|}{ Pyralidae } \\
\hline Chrysauge sp.1 & 1 & nd & $\mathrm{r}$ & $\mathrm{pf}$ & $\mathrm{z}$ & - & - & - & - & - & - & - & - & - & - \\
\hline Chrysauge sp. 2 & 1 & nd & $\mathrm{r}$ & $\mathrm{pf}$ & $\mathrm{z}$ & - & - & - & - & - & - & - & - & - & - \\
\hline Etiella zinckenella & - & - & - & - & - & - & - & - & - & - & 1 & nd & $\mathrm{r}$ & $\mathrm{pf}$ & $\mathrm{z}$ \\
\hline Milgithea argentilinea & 30 & $\mathrm{~d}$ & ma & $\mathrm{mf}$ & $\mathrm{y}$ & 21 & $\mathrm{~d}$ & $\mathrm{ma}$ & $\mathrm{mf}$ & $\mathrm{y}$ & 8 & $\mathrm{~d}$ & ma & $\mathrm{mf}$ & $\mathrm{y}$ \\
\hline Milgithea sp. & - & - & - & - & - & 1 & nd & $\mathrm{r}$ & $\mathrm{pf}$ & $\mathrm{Z}$ & - & - & - & - & - \\
\hline
\end{tabular}

D: Dominância - (sd) super dominante; (d) dominante; (nd) não dominante. A: Abundância - (sa) super abundante; (ma) muito abundante; (a) abundante; (c) comum; (d) dispersa; (r) rara. F: Frequência - (sf) super frequente; (mf) muito frequente; (f) frequente; (pf) pouco frequente. C: Constância - (w) constante; (y) acessória; (z) acidental. 
Análise faunística de lepidópteros em comunidades naturais no município de Cotriguaçu-MT, Brasil

\begin{tabular}{|c|c|c|c|c|c|c|c|c|c|c|c|c|c|c|c|}
\hline \multirow{2}{*}{$\begin{array}{l}\text { Família/Gênero/ } \\
\text { Espécie }\end{array}$} & \multicolumn{5}{|c|}{ Floresta nativa } & \multicolumn{5}{|c|}{ Mata ripária } & \multicolumn{5}{|c|}{ Capoeira } \\
\hline & $\begin{array}{c}\mathrm{N}^{\circ} \mathrm{de} \\
\text { Indivíduos }\end{array}$ & $\mathrm{D}$ & A & $\mathrm{F}$ & $\mathrm{C}$ & $\begin{array}{c}\mathrm{N}^{\circ} \mathrm{de} \\
\text { Indivíduos }\end{array}$ & $\mathrm{D}$ & A & $\mathrm{F}$ & $\mathrm{C}$ & $\begin{array}{c}\mathrm{N}^{\circ} \mathrm{de} \\
\text { Indivíduos }\end{array}$ & $\mathrm{D}$ & A & $\mathrm{F}$ & $\mathrm{C}$ \\
\hline Semnia sp.1 & 1 & nd & $\mathrm{r}$ & $\mathrm{pf}$ & $\mathrm{z}$ & - & - & - & - & - & - & - & - & - & - \\
\hline Semnia sp.2 & - & - & - & - & - & - & - & - & - & - & 1 & nd & $\mathrm{r}$ & $\mathrm{pf}$ & $\mathrm{z}$ \\
\hline Semnia subauritalis & 1 & nd & $\mathrm{r}$ & $\mathrm{pf}$ & $\mathrm{z}$ & 2 & nd & d & $\mathrm{pf}$ & $\mathrm{z}$ & 1 & nd & $\mathrm{r}$ & $\mathrm{pf}$ & $\mathrm{z}$ \\
\hline \multicolumn{16}{|l|}{ Riodinidae } \\
\hline Ancyluris aulestes & - & - & - & - & - & 1 & nd & $\mathrm{r}$ & $\mathrm{pf}$ & $\mathrm{Z}$ & - & - & - & - & - \\
\hline \multicolumn{16}{|l|}{ Saturniidae } \\
\hline Adelowalkeria sp.1 & 1 & nd & $\mathrm{r}$ & $\mathrm{pf}$ & $\mathrm{z}$ & - & - & - & - & - & 3 & nd & $\mathrm{c}$ & $\mathrm{f}$ & $\mathrm{z}$ \\
\hline Adelowalkeria sp.2 & 6 & $\mathrm{~d}$ & $\mathrm{c}$ & $\mathrm{f}$ & $\mathrm{y}$ & - & - & - & - & - & 3 & nd & $\mathrm{c}$ & $\mathrm{f}$ & $\mathrm{z}$ \\
\hline Automeris sp. & 7 & $\mathrm{~d}$ & ma & $\mathrm{mf}$ & $\mathrm{y}$ & - & - & - & - & - & - & - & - & - & - \\
\hline Caio sp. & - & - & - & - & - & 1 & nd & $\mathrm{r}$ & $\mathrm{pf}$ & $\mathrm{Z}$ & 2 & nd & $\mathrm{c}$ & $\mathrm{f}$ & $\mathrm{z}$ \\
\hline Cerodirphia mota napoensis & 5 & $\mathrm{~d}$ & $\mathrm{c}$ & $\mathrm{f}$ & $\mathrm{z}$ & - & - & - & - & - & - & - & - & - & - \\
\hline Citioica anthonilis & 4 & nd & $\mathrm{c}$ & $\mathrm{f}$ & $\mathrm{z}$ & 2 & nd & $\mathrm{d}$ & $\mathrm{pf}$ & $\mathrm{Z}$ & 2 & nd & $\mathrm{c}$ & $\mathrm{f}$ & $\mathrm{z}$ \\
\hline Citioica sp. & 2 & nd & $\mathrm{r}$ & $\mathrm{pf}$ & $\mathrm{z}$ & - & - & - & - & - & - & - & - & - & - \\
\hline Dirphia fraterna & 3 & nd & c & $\mathrm{f}$ & $\mathrm{z}$ & 2 & nd & d & $\mathrm{pf}$ & $\mathrm{Z}$ & 1 & nd & $\mathrm{r}$ & $\mathrm{pf}$ & $\mathrm{z}$ \\
\hline Dirphia sp.1 & 1 & nd & $\mathrm{r}$ & $\mathrm{pf}$ & $\mathrm{z}$ & - & - & - & - & - & 1 & nd & $\mathrm{r}$ & $\mathrm{pf}$ & $\mathrm{z}$ \\
\hline Dirphia sp.2 & 4 & nd & $\mathrm{c}$ & f & $\mathrm{y}$ & 1 & nd & $\mathrm{r}$ & $\mathrm{pf}$ & $\mathrm{Z}$ & 2 & nd & $\mathrm{c}$ & $\mathrm{f}$ & $\mathrm{z}$ \\
\hline Eacles imperialis magnifica & 2 & nd & $\mathrm{r}$ & $\mathrm{pf}$ & $\mathrm{z}$ & - & - & - & - & - & - & - & - & - & - \\
\hline Hylesia sp.1 & 5 & $\mathrm{~d}$ & $\mathrm{c}$ & $f$ & $\mathrm{z}$ & 2 & nd & $\mathrm{d}$ & $\mathrm{pf}$ & $\mathrm{z}$ & - & - & - & - & - \\
\hline Hylesia sp.2 & 61 & $\mathrm{~d}$ & $\mathrm{ma}$ & $\mathrm{mf}$ & $\mathrm{w}$ & 43 & $\mathrm{~d}$ & ma & $\mathrm{mf}$ & $\mathrm{w}$ & 26 & $\mathrm{~d}$ & ma & $\mathrm{mf}$ & $\mathrm{y}$ \\
\hline Hylesia sp.3 & 1 & nd & $\mathrm{r}$ & $\mathrm{pf}$ & $\mathrm{z}$ & 1 & nd & $\mathrm{r}$ & $\mathrm{pf}$ & $\mathrm{z}$ & 1 & nd & $\mathrm{r}$ & $\mathrm{pf}$ & $\mathrm{z}$ \\
\hline Hylesia sp.4 & 4 & nd & $\mathrm{c}$ & $\mathrm{f}$ & $\mathrm{z}$ & - & - & - & - & - & - & - & - & - & - \\
\hline Oiticella brevis & 2 & nd & $\mathrm{r}$ & $\mathrm{pf}$ & $\mathrm{z}$ & 1 & nd & $\mathrm{r}$ & $\mathrm{pf}$ & $\mathrm{z}$ & 1 & nd & $\mathrm{r}$ & $\mathrm{pf}$ & $\mathrm{z}$ \\
\hline Periga sp. & 1 & nd & $\mathrm{r}$ & $\mathrm{pf}$ & $\mathrm{z}$ & - & - & - & - & - & - & - & - & - & - \\
\hline Pseudoautomeris luteata & 1 & nd & $\mathrm{r}$ & $\mathrm{pf}$ & $\mathrm{z}$ & - & - & - & - & - & - & - & - & - & - \\
\hline
\end{tabular}

D: Dominância - (sd) super dominante; (d) dominante; (nd) não dominante. A: Abundância - (sa) super abundante; (ma) muito abundante; (a) abundante; (c) comum; (d) dispersa; (r) rara. F: Frequência - (sf) super frequente; (mf) muito frequente; (f) frequente; (pf) pouco frequente. C: Constância - (w) constante; (y) acessória; (z) acidental.

Continua... 


\begin{tabular}{|c|c|c|c|c|c|c|c|c|c|c|c|c|c|c|c|}
\hline \multirow{2}{*}{$\begin{array}{l}\text { Família/Gênero/ } \\
\text { Espécie }\end{array}$} & \multicolumn{5}{|c|}{ Floresta nativa } & \multicolumn{5}{|c|}{ Mata ripária } & \multicolumn{5}{|c|}{ Capoeira } \\
\hline & $\begin{array}{c}\mathrm{N}^{\circ} \mathrm{de} \\
\text { Indivíduos }\end{array}$ & $\mathrm{D}$ & A & $\mathrm{F}$ & $\mathrm{C}$ & $\begin{array}{c}\mathrm{N}^{\circ} \mathrm{de} \\
\text { Indivíduos }\end{array}$ & $\mathrm{D}$ & A & $\mathrm{F}$ & $\mathrm{C}$ & $\begin{array}{c}\mathrm{N}^{\circ} \mathrm{de} \\
\text { Indivíduos }\end{array}$ & $\mathrm{D}$ & A & $\mathrm{F}$ & $\mathrm{C}$ \\
\hline Rhescynthis hippodamia hippodamia & 4 & nd & $\mathrm{c}$ & $f$ & $\mathrm{y}$ & 1 & nd & $\mathrm{r}$ & $\mathrm{pf}$ & $\mathrm{z}$ & - & - & - & - & - \\
\hline Rothschildia erycina erycina & 3 & nd & $\mathrm{c}$ & $\mathrm{f}$ & $\mathrm{z}$ & - & - & - & - & - & 1 & nd & $\mathrm{r}$ & $\mathrm{pf}$ & $\mathrm{z}$ \\
\hline \multicolumn{16}{|l|}{ Sphingidae } \\
\hline Enyo ocypete & 1 & nd & $\mathrm{r}$ & $\mathrm{pf}$ & $\mathrm{z}$ & 1 & nd & $\mathrm{r}$ & $\mathrm{pf}$ & $\mathrm{z}$ & 1 & nd & $\mathrm{r}$ & $\mathrm{pf}$ & $\mathrm{z}$ \\
\hline Enyo sp. & - & - & - & - & - & 2 & nd & $\mathrm{d}$ & $\mathrm{pf}$ & $\mathrm{z}$ & - & - & - & - & - \\
\hline Erinnyis alope & 5 & $\mathrm{~d}$ & $\mathrm{c}$ & $\mathrm{f}$ & $\mathrm{y}$ & - & - & - & - & - & - & - & - & - & - \\
\hline Erinnyis oenotrus & 2 & nd & $\mathrm{r}$ & $\mathrm{pf}$ & $\mathrm{z}$ & - & - & - & - & - & 2 & nd & $\mathrm{c}$ & $\mathrm{f}$ & $\mathrm{z}$ \\
\hline Eumorpha anchemolus & 1 & nd & $\mathrm{r}$ & $\mathrm{pf}$ & $\mathrm{z}$ & - & - & - & - & - & 1 & nd & $\mathrm{r}$ & $\mathrm{pf}$ & $\mathrm{z}$ \\
\hline Madoryx sp. & - & - & - & - & - & - & - & - & - & - & 1 & nd & $\mathrm{r}$ & $\mathrm{pf}$ & $\mathrm{z}$ \\
\hline Manduca florestan & - & - & - & - & - & - & - & - & - & - & 1 & nd & $\mathrm{r}$ & $\mathrm{pf}$ & $\mathrm{z}$ \\
\hline Oryba achemenides & - & - & - & - & - & 1 & nd & $\mathrm{r}$ & $\mathrm{pf}$ & $\mathrm{z}$ & - & - & - & - & - \\
\hline Protambulyx strigilis & 1 & nd & $\mathrm{r}$ & $\mathrm{pf}$ & $\mathrm{z}$ & 1 & nd & $\mathrm{r}$ & $\mathrm{pf}$ & $\mathrm{z}$ & 3 & nd & $\mathrm{c}$ & $\mathrm{f}$ & $\mathrm{z}$ \\
\hline Protoparce sexta & - & - & - & - & - & - & - & - & - & - & 1 & nd & $\mathrm{r}$ & $\mathrm{pf}$ & $\mathrm{z}$ \\
\hline Pseudosphinx tetrio & 3 & nd & $\mathrm{c}$ & $\mathrm{f}$ & $\mathrm{y}$ & - & - & - & - & - & - & - & - & - & - \\
\hline Xylophanes chiron nechus & 1 & nd & $\mathrm{r}$ & $\mathrm{pf}$ & $\mathrm{z}$ & - & - & - & - & - & - & - & - & - & - \\
\hline Xylophanes porcus continentalis & 1 & nd & $\mathrm{r}$ & $\mathrm{pf}$ & $\mathrm{z}$ & - & - & - & - & - & - & - & - & - & - \\
\hline \multicolumn{16}{|l|}{ Thyrididae } \\
\hline Macrogonia sp. & 2 & nd & $\mathrm{r}$ & $\mathrm{pf}$ & $\mathrm{z}$ & 3 & nd & $\mathrm{c}$ & $\mathrm{f}$ & $\mathrm{y}$ & 1 & nd & $\mathrm{r}$ & $\mathrm{pf}$ & $\mathrm{z}$ \\
\hline \multicolumn{16}{|l|}{ Tineidae } \\
\hline Tiquadra aeneonivella & - & - & - & - & - & 1 & nd & $\mathrm{r}$ & $\mathrm{pf}$ & $\mathrm{Z}$ & - & - & - & - & - \\
\hline Tiquadra inscitella & - & - & - & - & - & 1 & nd & $\mathrm{r}$ & $\mathrm{pf}$ & $\mathrm{z}$ & - & - & - & - & - \\
\hline Tiquadra sp.1 & 1 & nd & $\mathrm{r}$ & $\mathrm{pf}$ & $\mathrm{z}$ & 1 & nd & $\mathrm{r}$ & $\mathrm{pf}$ & $\mathrm{z}$ & - & - & - & - & - \\
\hline Tiquadra sp.2 & 1 & nd & $\mathrm{r}$ & $\mathrm{pf}$ & $\mathrm{z}$ & 1 & nd & $\mathrm{r}$ & $\mathrm{pf}$ & $\mathrm{Z}$ & 2 & nd & $\mathrm{c}$ & $\mathrm{f}$ & $\mathrm{z}$ \\
\hline
\end{tabular}

D: Dominância - (sd) super dominante; (d) dominante; (nd) não dominante. A: Abundância - (sa) super abundante; (ma) muito abundante; (a) abundante; (c) comum; (d) dispersa; (r) rara. F: Frequência - (sf) super frequente; (mf) muito frequente; (f) frequente; (pf) pouco frequente. C: Constância - (w) constante; (y) acessória; (z) acidental. 
Análise faunística de lepidópteros em comunidades naturais no município de Cotriguaçu-MT, Brasil

Tabela 1. Continuação...

\begin{tabular}{|c|c|c|c|c|c|c|c|c|c|c|c|c|c|c|c|c|}
\hline \multirow{2}{*}{\multicolumn{2}{|c|}{$\begin{array}{l}\text { Família/Gênero/ } \\
\text { Espécie }\end{array}$}} & \multicolumn{5}{|c|}{ Floresta nativa } & \multicolumn{5}{|c|}{ Mata ripária } & \multicolumn{5}{|c|}{ Capoeira } \\
\hline & & $\begin{array}{c}\mathrm{N}^{\circ} \mathrm{de} \\
\text { Indivíduos }\end{array}$ & $\mathrm{D}$ & A & $\mathrm{F}$ & $\mathrm{C}$ & $\begin{array}{c}\mathrm{N}^{\circ} \text { de } \\
\text { Indivíduos }\end{array}$ & $\mathrm{D}$ & A & $\mathrm{F}$ & $\mathrm{C}$ & $\begin{array}{c}\mathrm{N}^{\circ} \text { de } \\
\text { Indivíduos }\end{array}$ & $\mathrm{D}$ & A & $\mathrm{F}$ & $\mathrm{C}$ \\
\hline \multicolumn{17}{|l|}{ Uraniidae } \\
\hline Urania leilus & & 2 & nd & $\mathrm{r}$ & $\mathrm{pf}$ & $\mathrm{z}$ & - & - & - & - & - & - & - & - & - & - \\
\hline \multicolumn{17}{|l|}{ Urodidae } \\
\hline Urodus sp. & & 4 & nd & $\mathrm{c}$ & $\mathrm{f}$ & $\mathrm{y}$ & 3 & nd & $\mathrm{r}$ & $\mathrm{pf}$ & $\mathrm{z}$ & - & - & - & - & - \\
\hline Total de indivíduos & & 747 & & & & & 463 & & & & & 348 & & & & \\
\hline Total de espécies & & 170 & & & & & 113 & & & & & 107 & & & & \\
\hline Índice de Diversidade & $\Rightarrow \mathrm{H}$ & 4,0876 & & & & & 3,7331 & & & & & 3,7694 & & & & \\
\hline
\end{tabular}

D: Dominância - (sd) super dominante; (d) dominante; (nd) não dominante. A: Abundância - (sa) super abundante; (ma) muito abundante; (a) abundante; (c) comum; (d) dispersa; (r) rara. F: Frequência - (sf) super frequente; (mf) muito frequente; (f) frequente; (pf) pouco frequente. C: Constância - (w) constante; (y) acessória; (z) acidental. 
Ecologicamente, a zona ripária é observada como corredor ecológico para a movimentação da fauna e dispersão dos vegetais, possibilitando o fluxo gênico in situ e ex situ (LIMA \& ZAKIA, 2000).

Em relação à quantidade de espécies a capoeira foi semelhante a mata ripária, fato esse que justifica seu papel positivo servindo como corredor para o translocamento da fauna e melhorando a conectividade.

Dentre as 26 famílias encontradas, Erebidae se destacou por apresentar maior quantidade de espécies (74) e indivíduos coletados (444), tendo maior ocorrência na floresta nativa (249). A maior parte dos Erebidae apresenta grande capacidade de dispersão (HILT, 2005). Ademais a maioria das espécies desta família são polífagas (DINIZ et al., 2001). Portanto, a associação entre corredor ecológico, alta capacidade de locomoção e a falta de restrição alimentar proporcionaria aos Erebidae a colonização de vários ambientes.

As espécies de maior representatividade quantitativa foram Paracles sp. 2 com 111 indivíduos em floresta nativa, Maruca vitrata com 63 indivíduos em mata ripária e Loxostege sp. 2 com 47 indivíduos em capoeira. Silva (2009), em estudo de diversidade de insetos em diferentes ambientes florestais em Cotriguaçu, registrou entre os lepidópteros coletados em área de floresta indivíduos de Paracles, no entanto não em quantidade expressiva. Segundo Dorval (1995), influências climáticas podem afetar diretamente (desenvolvimento, comportamento) e indiretamente (alimentação e ação de inimigos naturais) as populações.

Houve maior diversidade em floresta nativa $\left(H^{\prime}=4,0876\right)$ em comparação com os demais ambientes, demonstrando que as condições ambientais dessa área estão ecologicamente mais equilibradas.

De acordo com Andow (1991), a teoria ecológica sugere fatores importantes que levam a maior riqueza de espécies em locais mais heterogêneos, tendo estes a maior diversidade de habitats e a maior densidade de inimigos naturais, levando ao aumento do controle de populações e organismos dominantes.

Foram encontradas na floresta nativa 32 espécies dominantes, 138 não dominantes, 6 espécies constantes, 31 acessórias e 135 acidentais. Mata ripária apresentou 16 espécies dominantes, 97 não dominantes, 2 espécies constantes, 15 acessórias e 96 espécies acidentais. Na capoeira foram encontradas 16 espécies dominantes, 91 não dominantes, 1 constante, 11 acessórias e 95 espécies acidentais.

De acordo com Silveira Neto et al. (1976), os insetos dominantes têm capacidade de modificar um impacto recebido do ambiente em benefício próprio e dessa forma podem causar o aparecimento ou desaparecimento de outros organismos.

Segundo Clemente (1995), a alta percentagem de espécies acidentais, em comunidades florestais, indica resistência do meio à proliferação destas espécies.

Houve similaridade entre os ambientes área de capoeira e mata ripária, enquanto floresta nativa demonstrou maior dissimilaridade, em relação a distribuição dos indivíduos (Figura 1). De acordo com a análise, são similares quantitativamente, fato esse que demonstra a existência de condições semelhantes nessas áreas para o desenvolvimento das comunidades de insetos. 
Distância euclidiana

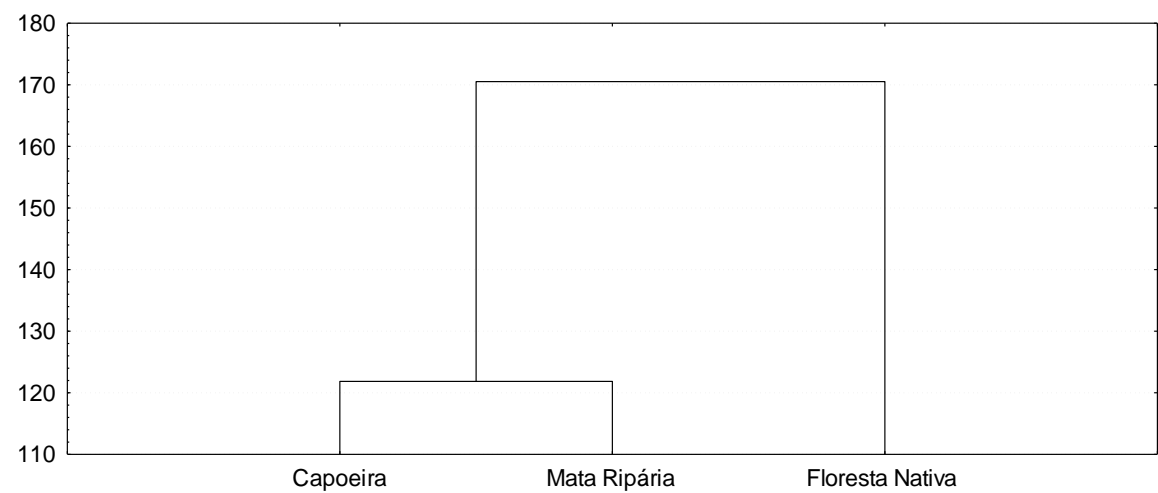

Figura 1. Dendrograma do estudo de similaridade entre os ambientes capoeira, mata ripária e floresta nativa no município de Cotriguaçu-MT.

\section{CONCLUSÕES}

A floresta nativa apresentou maior quantidade de espécies coletadas com maior índice de diversidade.

$\mathrm{Na}$ classificação de comunidades houve similaridade nos ambientes capoeira e mata ripária, com dissimilaridade no ambiente de floresta nativa .

Maruca vitrata e Hylesia sp. 2 foram as espécies dominantes e constantes em floresta nativa e mata ripária. Semiothisa sp.3 apresentou dominância e constância em floresta nativa e capoeira, sendo Paracles sp.1, Synchlora sp.2 e Macrosoma sp. as espécies que se destacaram como dominantes e constantes somente em floresta nativa.

\section{AGRADECIMENTOS}

A "Organización Nacionale du Fôret" (ONF/Brasil) por ter cedido o local para a realização da pesquisa, ao Prof. Dr. Sinval Silveira Neto da Escola Superior de Agricultura "Luiz de Queiroz" (ESALQ), da Universidade de São Paulo (USP) pela identificação das espécies e ao técnico de Laboratório (Faculdade de Engenharia Florestal/UFMT) Manoel Lauro da Silva pelo auxílio na condução desta pesquisa.

\section{REFERÊNCIAS BIBLIOGRÁFICAS}

ANDOW, D.A. 1991. Vegetational diversity and arthropod population response. Annual review of entomology, Palo Alto, v. 35, p.561-586

BRASIL. 1982. Ministério das Minas e Energia. Departamento de Produção Mineral. Projeto RADAMBRASIL. Levantamento de Recursos Naturais, v. 20, folha SC 21, Juruena, MT.

CAMARGO, A.J.A. 1999. Estudo comparativo sobre a composição e a diversidade de lepidópteros noturnos em cinco áreas da Região dos Cerrados. Revista Brasileira de Zoologia, Curitiba, v. 16, n. 2, p. 369-380

CLEMENTE, A.T.C. 1995. Análise de populações de Lepidoptera em comunidades florestais de Araucaria angustifolia, Eucalyptus grandis e Pinus taeda. Curitiba: UFPR, 1995, 75 p. Dissertação. (Mestrado) Universidade Federal do Paraná.

DAJOZ, R. 1973. Ecologia geral. Petrópolis, EDUSP. Vozes, 474 p.

DINIZ, I.R.; MORAIS, H.C.; CAMARGO, A.J.A. 2001. Hosts plants of lepidopteran caterpillars in the Cerrado of the Distrito Federal, Brasil. Revista Brasileira de Entomologia, v.45, p-107-122 
DORVAL, A. 1995. Análise faunística e flutuação populacional de lepidópteros em Eucalyptus urophylla e Eucalyptus cloeziana em Montes Claros, MG. Viçosa. 1995. 80p. Dissertação (Mestrado em Entomologia) Universidade Federal de Viçosa, ViçosaMG.

FERREIRA, P.SF; MARTINS, D.S. 1982. Contribuição ao método de captura de insetos por meio de armadilha luminosa, para obtenção de exemplares sem danos morfológicos. Revista Ceres, v.29, p.538-543

FREITAS, A.V.L.; FRANCINI, R., BROWN JR., K.S. 2003. Insetos como indicadores ambientais. In: CULLEN JR., L. RUDRAN, R.; VALADARESPADUA, C. (Ed.). Manual Brasileiro em Biologia da Conservação. Curitiba: Editora da UFPR. Fundação O Boticário de Proteção à Natureza, p. 125-148

HILL, M.O. 1973. Diversity and evenness: a unifying notation and its consequences. Ecology, Ithaca, v. 54, p. 427-431

HILT, N. 2005. Diversity and species composition of two different moth families (Lepidoptera: Arctiidae vs. Geometridae) along successional gradient in the Ecuadorian Andes. 2005. 251p. Tese de doutorado Universidade de Bayreuth. Alemanha.

JOST, L. 2006. Entropy and diversity. Oikos, Lund, v. 113, n. 2, p. 363-375

LIMA, W.P.; ZAKIA, M.J.B. 2000. Hidrologia de matas ciliares. In: RODRIGUES, R.R.; LEITÃO FILHO, H.F. ed. Matas ciliares: conservação e recuperação. São Paulo: EDUSP/ Fapesp, cap.3, p.33-44

MARINONI, R.C.; DUTRA, R.R.C.; MIELKE, O.H.H. 1999. Levantamento da fauna entomológica no Estado do Paraná. IV. Sphingidae (Lepidoptera). Diversidade alfa e estrutura de comunidade. Revista Brasileira de
Zoologia, Curitiba, v. 16, (Supl. 2), p. 223-240

MOTTA, P.C. 2002. Butterflies from the Uberlândia region, central Brazil: species list and biological comments. Brazilian Journal of Biology, São Carlos, v.62, n.1, p.151-163

PRICE, P.W. 1975. Insect ecology. New York: J. Wiley, 514 p.

SAKAGAMI, S.F.; LAROCA, S. 1967. Observations on the bionomics of some neotropical Xylocopini bees, with some comparative biofaunistic notes (Hymenoptera, Anthophoridae). Jour. Fac. Sci., Hokkaido, v.18, p.57-127

SANTOS, G.P.; ZANUNCIO, T.V.; VINHA, E.; ZANUNCIO, J.C. 2002. Influência de faixas de vegetação nativa em povoamentos Eucalyptus cloeziana sobre população de Oxydia vesulia (Lepidoptera: Geometridae). Revista Árvore, Viçosa, v. 26, p. 499-504

SILVA, M. M. 2009. Diversidade de insetos em diferentes ambientes florestais no município de Cotriguaçu, estado de Mato Grosso. Cuiabá. 2009. 48p. Dissertação (Mestrado em Ciências Florestais e Ambientais) - Universidade Federal de Mato Grosso, Cuiabá-MT.

SILVEIRA NETO, S.; NAKANO, O.; BARBIN, D.; VILLA NOVA, N.A. 1976. Manual de ecologia dos insetos. Piracicaba: Ceres, 419 p.

SILVEIRA NETO, S.; SILVEIRA, A.C. 1969. Armadilha luminosa modelo "Luiz de Queiroz". O Solo. Piracicaba, v.61, n.2, p.19-21

WOOD, B.; GILMAN, M.P. 1998. The effects of disturbance on forest butterflies using two methods of sampling in Trinidad. Biodiversity and Conservation, vol.7, p.597-616.

Recebido em: 17/05/2012 Aceito para publicação em: 19/08/2013 